\title{
Activation of the Hedgehog and Wnt/ $\beta$-Catenin Signaling Pathways in Basal Cell Carcinoma
}

\author{
Tomoaki Takada \\ Sumikawa Takada Dermatology Clinic, Sapporo, Japan
}

\section{Keywords}

Basal cell carcinoma · Glioma-associated oncogene 1 - Smoothened · Insulin-like growth factor 2 mRNA-binding protein 1

\begin{abstract}
In basal cell carcinoma (BCC) tumorigenesis, interaction between Hedgehog (Hh) and Wnt/ $\beta$-catenin (Wnt) signaling pathways has been investigated, but not completely elucidated. Here, a case of sporadic BCC in an 80-year-old man is presented, and the effectiveness of SMO inhibitors in case of relapse is predicted. The aim of this study was to determine whether the SMO inhibitors can be effective in treating this individual should the tumor recur in the future. Immunohistochemistry (IHC) was performed in a tumor and the adjacent skin tissue from the patient. IHC within the same BCC tissue specimen revealed that Glioma-associated oncogene 1 (GLI1) and Smoothened (SMO) in the Hh signaling pathway and insulin-like growth factor 2 mRNA-binding protein 1 (IGF2BP1) in the Wnt signaling pathway were overexpressed. Hh and Wnt signaling pathways were activated. These findings suggest that the patient might be resistant to treatment with SMO inhibitors because of the interaction between $\mathrm{Hh}$ and Wnt signaling pathways. Overexpression of GLI1 leads to transcriptional activation, making it an attractive molecular target for anticancer therapy owing to the downstream effectors of the cascade.
\end{abstract}

\section{Karger $\stackrel{N}{\%}$}




\section{Introduction}

BCC is the most common human cancer, characterized by aberrant activation of the $\mathrm{Hh}$ signaling pathway resulting from mutations in the patched 1 (PTCH1) or SMO genes [1]. Sporadic BCCs consistently express glioma-associated oncogene 1 (GLI1), which acts as a target and mediator of the Sonic hedgehog (Shh) pathway. Any mutation leading to GLI expression in basal cells has been predicted to induce BCC formation [2]. The Wnt signaling pathway stimulates the transcriptional output of $\mathrm{Hh}$ signaling. Wht signaling induces the expression of RNA-coding region determinant binding protein (CRD-BP) or insulin-like growth factor $2 \mathrm{mRNA}$-binding protein 1 (IGF2BP1), which in turn binds and stabilizes GLI1 mRNA, thereby upregulating GLI1 expression and transcriptional activity [3, 4]. Currently, the Food and Drug Administration (FDA)-approved Hh pathway drugs include the SMO inhibitors vismodegib and sonidegib [5]. In the present study, immunohistochemistry (IHC) was performed to analyze the expression patterns of GLI1, SMO, and IGF2BP1 in a tumor and the tumor adjacent tissue from a patient with sporadic BCC. The aim of this study was to determine whether the SMO inhibitors can be effective in treating this individual should the tumor recur in the future.

\section{Case Presentation}

\section{Case History and Examination}

An 80-year-old man presented with a "ca. $5 \mathrm{~mm} \times 4 \mathrm{~mm}$ " waxy black spot on the left cheek over 1 year ago. The limbus was slightly irregular, whereas the concavity and convexity of the surface were recognized. The dermoscopy (DZ-100; Yamagata Casio Co., Ltd., Higashine, Japan) showed the presence of leaf-like structures and multiple blue-gray globules. Few findings in dermoscopy were suggestive of melanocytic and vascular lesions (Fig. 1a). Histopathological examination by hematoxylin and eosin (H\&E) staining showed typical tumor nests of BCC, an elevated lesion with slight ulceration and crusting in the epidermis, hair blast-like cells with densely stained nuclei, and a few cytoplasmic organelles showed a nodular or cord-like growth pattern. The nuclei in the margins of tumor nests presented a palisade arrangement. Alcian blue staining revealed mucin deposition in the surrounding dermal stroma around tumor nests. Some of the intradermal tumors showed continuity with the epidermis (Fig. 2).

\section{Differential Diagnosis, Investigation, and Treatment}

The patient was diagnosed with BCC, and a surgical resection with flap creation of the affected tissue was performed with a 4-mm safety margin (Fig. 1b). Written informed consent was obtained from the patient for the use of the tissue samples in this study. The final diagnosis was BCC, solid type. IHC was performed with rabbit polyclonal anti-GLI1 (ab217326; Abcam, Cambridge, UK), rabbit polyclonal anti-SMO (20787-1-AP; ProteinTech, Rosemont, IL, USA), and rabbit polyclonal anti-IGF2BP1 (22803-1-AP; ProteinTech) antibodies. The staining evaluation was performed using the standard scores of the World Health Organization: $<20 \%$ (nucleus) or $<25 \%$ (cytoplasm) stained cells indicated negative/low expression; $20-50 \%$ (nucleus) or $25-50 \%$ (cytoplasm) staining indicated moderate expression; and $>50 \%$ (nucleus or cytoplasm) staining indicated high expression. GLI1 expression was high in both the nucleus and cytoplasm of the tumor tissue cells. In the adjacent normal skin tissue, GLI1 expression was high in the nucleus but low in the cytoplasm. SMO expression showed trends similar to GLI1 in the tumor cells as well as the adjacent skin tissue. IGF2BP1 showed high expression in both the nucleus and cytoplasm of tumor cells and moderate expression in the adjacent skin tissue (Fig. 3b-d).

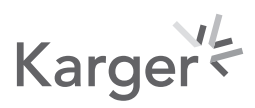



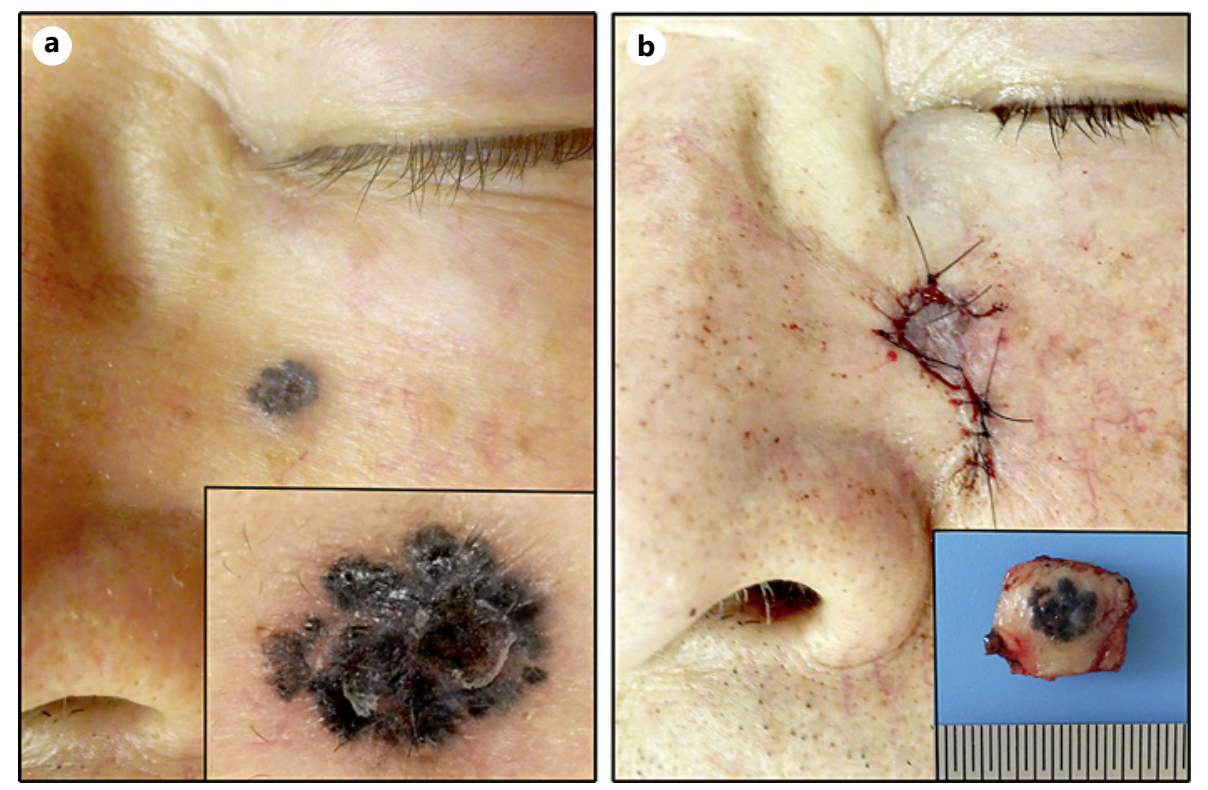

Fig. 1. Clinical presentation. a Nodular basal cell carcinoma on the left cheek. Dermoscopy showed leaf-like structures and multiple blue-gray globules (inset outlined with a black box). b Postoperative clinical photograph. Surgical resection with flap creation was performed. The resected specimen with a 4-mm margin of the normal tissue (inset outlined with a black box).

Fig. 2. Histology findings. a Nodular tumor aggregates may be of varying sizes. Nodular basal cell carcinoma, the most common type, generally consists of large, round, or oval tumor islands within the dermis, often with an epidermal attachment. The peripheral cell mass is in a palisade arrangement that resembles the basal layer of the epidermis. The connective tissue stroma surrounding the tumor islands is arranged in parallel bundles. Melanin is also present within the tumor and the surrounding stroma. Increased mucin is present in the surrounding dermal stroma. Hematoxylin-eosin (H\&E) staining. Original magnification, $\times 30$; scale bar, $500 \mu \mathrm{m}$. Inset (black box): $\times 400$; scale bar, $50 \mu \mathrm{m}$. b Mucin deposition can be seen as blue color around the tumor. Alcian blue staining. Original magnification, $\times 30$; scale bar, $500 \mu \mathrm{m}$.
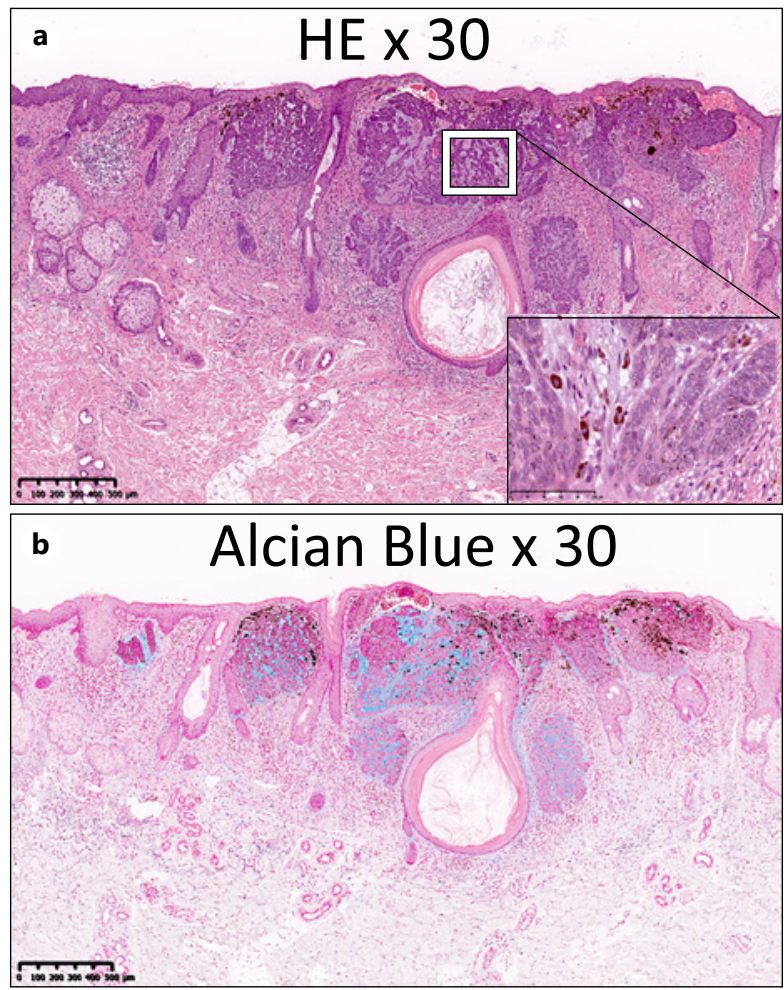

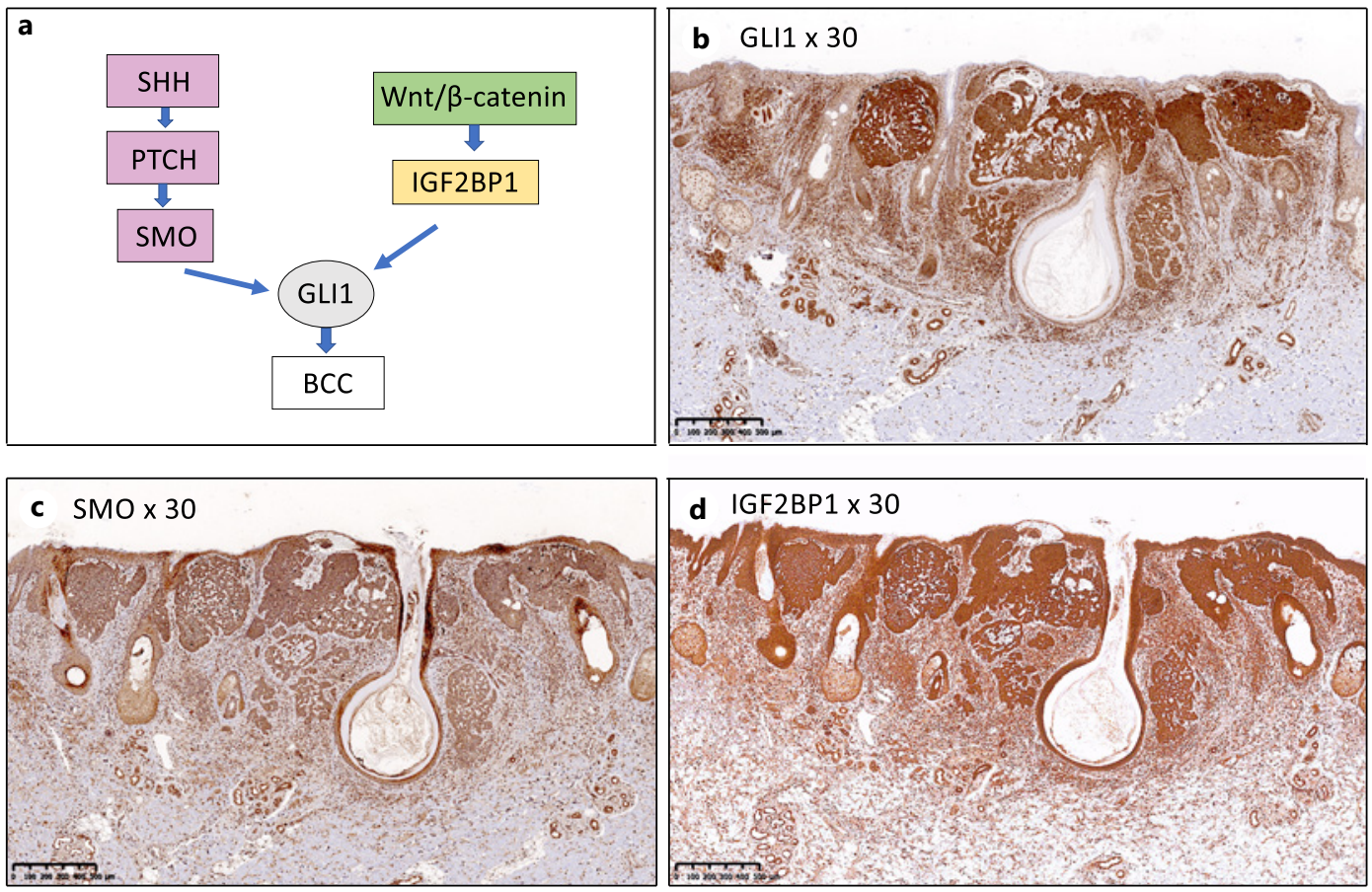

Fig. 3. Crosstalk between Hh and Wnt signaling pathways and immunohistochemical staining of GLI1, SMO, and IGF2BP1. a Smo activation leads to GLI1 transcriptional activation and upregulation of IGF2BP1. Wnt and $\mathrm{Hh}$ are major pathways to interact at multiple levels. Wnt induces the expression and transcriptional activity of GLI1 in an IGF2BP1-dependent manner. b Immunohistochemical staining of GLI1 expression was high in both the nucleus and cytoplasm of the tumor cells. In the adjacent normal skin cells, the expression was high in the nucleus and low in the cytoplasm. GLI1 staining. Original magnification, $\times 30$; scale bar, $500 \mu \mathrm{m}$. c SMO expression was high in both the nucleus and cytoplasm in the tumor tissue. In the adjacent normal skin cells, nuclear expression was high and cytoplasmic expression was low. SMO staining. Original magnification, $\times 30$; scale bar, $500 \mu \mathrm{m}$. d IGF2BP1 showed high expression in both the nucleus and cytoplasm of the tumor cells. In the adjacent normal skin cells, nuclear and cytoplasmic expression was low. IGF2BP1 staining. Original magnification, $\times 30$; scale bar, $500 \mu \mathrm{m}$.

\section{Outcome and Follow-Up}

The patient was finally diagnosed with BCC by histopathological analysis and a subsequent resection of the tumor tissue followed. There were no findings of recurrence 6 months after the operation, and postoperative follow-ups with regular intervals have been planned.

\section{Discussion}

BCC is the most common human cancer worldwide and is a subtype of nonmelanoma skin cancer [6]. Its incidence has been increasing consistently because of the aging population and exposure to radiations from the sun. Although the mortality rate associated with BCC is negligible, the morbidity and healthcare costs have risen significantly in recent decades. BCC displays a different behavior compared with other neoplasms; it has a slow evolution and metastasizes very rarely, but sometimes causes significant local damage. Chronic ultraviolet exposure, along with genetic factors, is the most important risk factor for the development of BCC [7]. Whole-exome sequencing to characterize the mutational landscape of sporadic BCCs 
has revealed that only Patched 1 (PTCH1) is associated with a significant functional mutation burden. These findings support the central role of PTCH1 mutations in BCC genesis [8]. Mutations in PTCH1 are associated with Gorlin syndrome, an autosomal dominant disorder characterized by the occurrence of multiple BCCs; they are also the most frequent mutations observed in sporadic BCCs. PTCH1 encodes the PTCH1 protein, the most important negative regulator of the Hh pathway. Numerous studies are being conducted to confirm the involvement of the Hh pathway in BCC pathogenesis. Although the Hh pathway has been intensively investigated, it has not been completely elucidated.

Activation of Hh appears to be a key driver of BCC development. The PTCH1 mutations play a crucial role in activating the $\mathrm{Hh}$ pathway; however, additional mutations that promote BCC carcinogenesis have been identified. Although activation of Hh appears to be a key driver of BCC development, recent studies on BCC tumorigenesis have shown that there are also other pathways involved in BCC development. For example, crosstalk between Hh and other signaling pathways, such as Wnt, EGFR, p53, PI3K/mTOR, and vitamin D, has been demonstrated [3, 7]. A further argument for the involvement of other pathways in the development of BCC could be the reason for tumor resistance to Hh pathway inhibitors (HhI) [7].

Studies involving mouse models have provided evidence that the activation of the GLI family of transcription factors is a key step in the initiation of the tumorigenic program leading to BCC. Expression of GLI1 in basal cells induces BCC formation [2]. The activation of the Wnt pathway is also observed in BCCs. In addition, the Wnt pathway was required for Hh-driven development of BCC in a mouse model. Crosstalk between the Wnt and Hh pathways has been observed at different levels, yet the mechanisms underlying this crosstalk are not fully understood [7]. Recent studies have identified IGF2BP1 (also known as IMPI1, CRD-BP, and ZBP1), a direct target of Wnt signaling, as a factor that binds to GLI1 mRNA and upregulates its levels and activity. This mode of regulation by GLI1 appears to be important in BCC tumorigenesis and could be explored in the treatment of BCCs [3]. A novel mechanism by which the Wnt signaling regulates the transcriptional outcome of the Hh signaling pathway has been identified. It has been demonstrated that IGF2BP1 binds to GLI1 mRNA, stabilizes it, and consequently upregulates its mRNA and protein levels and activities. It was hypothesized that Wntinduced and IGF2BP1-dependent regulation of GLI1 expression and activity are important for the development of BCC. IGF2BP1 is overexpressed in BCC, and its expression positively correlates with the activation of both Wnt and Hh pathways [4]. Another study also described the generation and characterization of a human BCC cell line to demonstrate the importance of IGF2BP1-dependent regulation of GLI1 expression and activity in the development of BCC [9]. These pieces of evidence from previous studies indicate why IGF2BP1 was the target of IHC in this case.

The primary goal of treating BCC is the complete removal of the tumor and maximal preservation of function and cosmesis. All treatment decisions should be customized to account for the particular factors present in the individual case and the patient's preference. Cemiplimab-rwlc was approved for patients with locally advanced or metastatic BCC previously treated with an $\mathrm{HhI}$ or for whom an $\mathrm{HhI}$ is not appropriate [5]. Programmed death-1 (PD-1) is a cell surface receptor that functions as a T-cell checkpoint and plays a central role in regulating T-cell exhaustion. Binding of PD-1 to its ligand, programmed death-ligand 1 (PD-L1), activates downstream signaling pathways and inhibits T-cell activation. Cemiplimabrwlc is a PD-1/PD-L1 immune checkpoint blockade [10].

$S H H$ and GLI are normally expressed in hair follicles, and sporadic BCCs consistently express GLI1. The expression of GLI1 in basal cells induces BCC formation. Any mutation leading to the expression of GLI1 in basal cells is predicted to induce BCC formation [2]. BCC is characterized by aberrant activation of the Hh pathway resulting from mutations in PTCH1

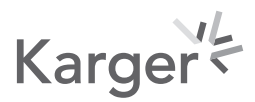


or SMO. The expression profile of Hh signaling-related molecules and the mRNA and protein expression levels of 6 molecules including GLI1, GLI2, PTCH1, PTCH2, SHH, and SMO in BCC and various other cutaneous tumors demonstrated that BCC shows remarkably enhanced mRNA expression of all Hh molecules, except SMO, compared with other skin tumors. IHC analysis revealed that only GLI1 was specifically upregulated in BCC, whereas the other Hh-related proteins did not show any significant differences between the tumors. There was no difference in GLI1 expression between BCC subtypes [1].

An interaction between the Hh and Wnt signaling pathways may be involved in the development of BCCs [1]. The Wnt and Hh pathways play central roles in embryogenesis, stem cell maintenance, and tumorigenesis. The Wnt pathway stimulates the transcriptional output of Hh signaling. Wht signaling induces the expression of the RNA-binding protein IGF2BP1, which in turn binds and stabilizes GLI1 mRNA, thereby upregulating GLI1 expression and transcriptional activity [4]. The stabilization of GLI1 mRNA by the coding region determinant binding protein, IGF2BP1, through the Wnt signaling pathway is implicated in the proliferation of colorectal cancer and BCC. A specific oligonucleotide was found to be effective in blocking IGF2BP1-GLI1 RNA interaction [11]. A cellular screen for small-molecule antagonists of GLI-mediated transcription, which constitutes the final step in the Hh pathway, revealed 2 molecules that can selectively inhibit GLI-mediated gene transactivation. Mechanistically, both inhibitors act in the nucleus to block GLI function, and one interferes with GLI1 DNAbinding in living cells [12].

Inhibitors targeting the Hh signal transducer SMO are widely used, and they display a good initial efficacy in patients with BCC; however, a large number of patients relapse. Although the $S M O$ mutations may explain acquired therapy resistance, a growing body of evidence suggests that the noncanonical, SMO-independent activation of the Hh pathway in patients with BCC can also account for this adverse effect. The importance of GLI transcription factors as the main downstream effectors of the canonical and noncanonical $\mathrm{Hh}$ cascade and their putative role in the regulation of multiple oncogenic signaling pathways have been highlighted [13]. Here, IHC within the same BCC tissue specimen revealed that GLI1 and SMO in the Hh signaling pathway and IGF2BP1 in the Wnt signaling pathway were overexpressed.

Understanding and characterizing the BCC signaling pathways and the interactions among them could help develop new therapies for BCC. Numerous lines of evidence support a therapeutic strategy with small-molecule antagonists of GLI1-mediated transcription constituting the final step in the Hh signaling pathway. The pathogenesis of basal cell carcinoma is highly complex. The positive IHC expression of SMO, GLI1, and IGF2BP1 in this case suggests numerous unknown interactions between multiple signaling pathways. $\mathrm{Hh}$ signaling pathway and other signaling pathways in the development of BCC could be the cause of tumor resistance to $\mathrm{HhI}$ [7]. Therefore, it is speculated that the patient might be resistant to treatment with SMO inhibitors in this case. Histone deacetylase (HDAC) inhibitors, remetiostat [14], may have promise in BCC, potentially through sequestering GLI to the inner nuclear membrane. These include small-molecular antagonists of the transcription factor downstream of the Hh pathway, GLI1, which may be able to avoid the issues of the resistance experienced by inhibitors acting upstream.

\section{Acknowledgments}

Genetic Lab Co., Ltd. provided helpful discussions. I am also grateful to the patient for providing consent for the use of his samples in this study. I thank Wiley Editing Service for English language editing.

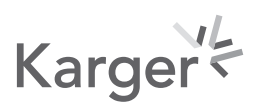


Case Reports in Dermatology

\begin{tabular}{l|l}
\hline Case Rep Dermatol 2021;13:506-512 \\
\hline DOI: 10.1159/000520256 & $\begin{array}{l}\text { @ 2021 The Author(s). Published by S. Karger AG, Basel } \\
\text { www.karger.com/cde }\end{array}$ \\
\hline
\end{tabular}

Takada: Hedgehog and Wnt/ $\beta$-Catenin Signaling in Basal Cell Carcinoma

\section{Statement of Ethics}

Written informed consent was obtained from the patient for publication of this case report and accompanying images. All procedures adopted in the present study were in respect to the ethical standards in the World Medical Association Declaration of Helsinki. Ethical approval was not required for this study in accordance with local/national guidelines.

\section{Conflict of Interest Statement}

The author has no conflicts of interest to declare.

\section{Funding Sources}

This study did not receive any funding.

\section{Data Availability Statement}

The datasets used and/or analyzed during the current study are available from the corresponding author upon reasonable request.

\section{References}

1 Kim HS, Kim YS, Lee C, Shin MS, Kim JW, Jang BG. Expression profile of sonic hedgehog signaling-related molecules in basal cell carcinoma. PLoS One. 2019 Nov;14(11):e0225511.

2 Dahmane N, Lee J, Robins P, Heller P, Ruiz i Altaba A. Activation of the transcription factor Gli1 and the Sonic hedgehog signalling pathway in skin tumours. Nature. 1997 Oct;389(6653):876-81.

3 Noubissi FK, Yedjou CG, Spiegelman VS, Tchounwou PB. Cross-talk between Wnt and Hh signaling pathways in the pathology of basal cell carcinoma. Int J Environ Res Public Health. 2018 July;15(7):1442.

4 Noubissi FK, Goswami S, Sanek NA, Kawakami K, Minamoto T, Moser A, et al. Wnt signaling stimulates transcriptional outcome of the Hedgehog pathway by stabilizing GLI1 mRNA. Cancer Res. 2009 Nov;69(22): 8572-8.

5 National Comprehensive Cancer Network. Clinical practice guidelines in oncology. Basal cell skin cancer version 2 [Internet]. 2021 [cited 2021 Mar 24]. Available from: https://www.nccn.org/professionals/ physician_gls/pdf/nmsc.pdf.

6 Verkouteren JAC, Ramdas KHR, Wakkee M, Nijsten T. Epidemiology of basal cell carcinoma: scholarly reviw. Br J Dermatol. 2017 Aug;177(2):359-72.

7 Tampa M, Georgescu SR, Mitran CI, Mitran MI, Matei C, Scheau C, et al. Recent advances in signaling pathways comprehension as carcinogenesis triggers in basal cell carcinoma. J Clin Med. 2020 Sep;9(9):3010.

8 Jayaraman SS, Rayhan DJ, Hazany S, Kolodney MS. Mutational landscape of basal cell carcinomas by wholeexome sequencing. J Invest Dermatol. 2014 Jan;134(1):213-20.

9 Noubissi FK, Kim T, Kawahara TN, Aughenbaugh WD, Berg E, Longley BJ, et al. Role of CRD-BP in the growth of human basal cell carcinoma cells. J Invest Dermatol. 2014 Jun;134(6):1718-24.

10 Jiang Y, Chen M, Nie H, Yuan Y. PD-1 and PD-L1 in cancer immunotherapy: clinical implications and future considerations. Hum Vaccin Immunother. 2019;15(5):1111-22.

11 Mehmood K, Akhtar D, Mackedenski S, Wang C, Lee CH. Inhibition of GLI1 expression by targeting the CRDBP-GLI1 mRNA interaction using a specific oligonucleotide. Mol Pharmacol. 2016 Jun;89(6):606-17.

12 Lauth M, Bergström A, Shimokawa T, Toftgård R. Inhibition of GLI-mediated transcription and tumor cell growth by small-molecule antagonists. Proc Natl Acad Sci U S A. 2007 May;104(20):8455-60.

13 Didiasova M, Schaefer L, Wygrecka M. Targeting GLI transcription factors in cancer. Molecules. 2018 Apr; 23(5):1003.

14 Kilgour JM, Shah A, Urman NM, Eichstadt S, Do HN, Bailey I, et al. Phase II open-label, single-arm trial to investigate the efficacy and safety of topical remetinostat gel in patients with basal cell carcinoma. Clin Cancer Res. 2021 Sep 1;27(17):4717-25. 